\title{
Detection of a single nucleotide polymorphism (SNP) DNA marker linked to cocoon traits in the mulberry silkworm, Bombyx mori (Lepidoptera: Bombycidae)
}

\author{
Sivaramakurup SREEKUMAR, Southekal K. ASHWATH*, Monika SLATHIA, Sundaramurthy N. KUMAR \\ and SYED M.H. QADRI
}

\begin{abstract}
Silkworm Genetics and Molecular Biology Laboratory, Central Sericultural Research and Training Institute, Srirampura, Mysore-570008, India; e-mail: ashwathsk@yahoo.com
\end{abstract}

Key words. Bombyx mori, bulked segregant analysis, cocoon traits, single nucleotide polymorphism, quantitative trait loci, QTL

\begin{abstract}
Cocoon weight and shell weight are the key economic traits ultimately determining silk yield. In order to detect the main quantitative trait loci (QTL) associated with the cocoon traits of the mulberry silkworm, Bombyx mori, the parents of larvae that produced cocoons that differed greatly in weight and shell weight were screened using 240 primer pairs of single nucleotide polymorphic markers (SNPs) representing all the 28 linkage groups in silkworm. Out of the 240 primer pairs, $48(20 \%)$ revealed distinct polymorphism between the parents, which was confirmed by the co-dominant expression of both polymorphic PCR products in the $F_{1}$ generation. The bulked segregant analysis (BSA) was used to compare the SNP profiles of the parents, $F_{1}$ and $F_{2}$ bulks using the 48 informative SNP primers. This revealed that out of 48 primer pairs, only one pair, i.e., No. 04124 of the linkage group 4 showed clear differences in the amplified products between the bulks corresponding to that of the parents with different cocoon traits suggesting that the DNA regions amplified by this primer pair are closely linked to the QTL controlling the cocoon traits. The results were also confirmed by screening the backcross (BC) progeny. This is the first report of the identification of a QTL using SNPs with BSA. The results of the present study indicate that it might be possible to use SNPs for marker assisted selection (MAS) in silkworm breeding programs aimed at improving cocoon traits.
\end{abstract}

\section{INTRODUCTION}

Though India is today the second largest producer of silk in the world, the twin problems of low productivity and poor fibre quality continue to impair an increase in production. To overcome these drawbacks, new breeding strategies adopted in the early ' 90 s have resulted in the production of a number of productive silkworm strains. However, these strains are popular only with those farmers who can provide adequate inputs and managerial skills. Even today, the bulk of the silk in India is produced by a polyvoltine $\times$ bivoltine cross strain and is not of the required International standard. As a result, India imports large quantities of silk from China. The available bivoltine strains that produce quality silk are not hardy. Hence the polyvoltine strains need to be improved by incorporating genes carrying the desired traits so that they produce high quality silk. Molecular markers associated with quantitative trait loci (QTL) are reported for many crops and many important traits. After linkage between QTL and a molecular marker is determined, the QTL can be transferred to a different genetic background by marker assisted selection.

For the identification of DNA markers closely linked to a selected character, one of the strategies adopted to identify the few that are tightly linked to the target gene from a large number of markers is bulked segregant analysis
(BSA). This technique was successfully used to identify DNA markers linked to the downy mildew resistance gene in lettuce (Michelmore et al., 1991), leaf rust resistance in barley (Poulsen et al., 1995), malaria and nematode resistance genes in mosquitoes (Severson et al., 1999), powdery mildew resistance gene in wheat (Chantret et al., 2000), sex determination in aphids (Gadau et al., 2001), vaccine response in chicks (Kaiser et al., 2002), QTL for grain quality in rice (Govindaraj et al., 2005), sex expression in shrimps (Khamnamtong et al., 2006), genetic mapping in zebra fish (Bradley et al., 2007), clubroot resistance genes in oilseed rape (Werner et al., 2008), red wing patterning in Heliconius butterflies (Baxter et al., 2008), isolation of sex-linked SCAR markers in yellow catfish (Wang et al., 2009), and powdery mildew resistance gene in soybean (Kang \& Mian, 2010).

With the goal of identifying DNA markers closely linked to the cocoon trait in mulberry silkworm, Bombyx mori, the present work was undertaken using parents with contrasting features for cocoon traits. SNP primers were screened between parents and the informative primers showing clear cut polymorphism between the parents were profiled among the parents, and $F_{1}$ and $F_{2}$ bulk samples. In addition, backcross (BC) progeny were also analyzed to confirm the results.

\footnotetext{
* Corresponding author.
} 


\section{MATERIAL AND METHODS}

Silkworm strains used and raising of $F_{2}$ and $B C$ progeny

The silkworm strains Pure Mysore (PM) and CSR2, which differ in cocoon traits, were used in the present study. The parents were reared under standard conditions and the $\mathrm{F}_{1}$ generation of $\mathrm{PM} \times \mathrm{CSR} 2, \mathrm{~F}_{2}$ by selfing of $\mathrm{F}_{1}$ progeny, backcross progeny, viz., $(\mathrm{PM} \times \mathrm{CSR} 2) \times \mathrm{PM}$ and $(\mathrm{PM} \times \mathrm{CSR} 2) \times \mathrm{CSR} 2$, were produced and reared simultaneously. After cocooning the cocoons were cut open and sexes of the pupae determined by recording the markings on the individual pupae. Cocoon weight represents the total weight of the pupa along with the silken shell. The shell weight is the weight of the cocoon after removing the pupa. Generally, female pupae are heavier than those of males, as they contain 350 to 500 eggs and a geater amount of fat tissue. To determine the mean cocoon weight of parents and progeny, a sample of 100 cocoons consisting of 50 female and 50 male cocoons of the parents, $\mathrm{F}_{1}, \mathrm{~F}_{2}$ and $\mathrm{BC}$ progeny were weighed. Further the shell weight was recorded using the same cocoon samples. The frequency distribution of the cocoon and shell weight of the $F_{2}$ was analyzed. The cocoon weight and shell weight data were sorted in descending order and the top ten (high weight) and the bottom ten (low weight) in the samples of $\mathrm{F}_{2}$ and $\mathrm{BC}$ progeny were selected.

\section{DNA extraction}

High molecular weight genomic DNA was extracted from frozen moths of PM females, CSR2 males, and their $F_{1}$ progeny $(\mathrm{PM} \times \mathrm{CSR} 2)$. Frozen pupae were used for the extraction of DNAs from $F_{2}$ and $\mathrm{BC}$ progenies. All DNA extractions were carried out using the standard phenol-chloroform method. The ethanol precipitated DNA was resuspended in TE buffer $(5 \mathrm{mM}$ Tris- $\mathrm{HCl}$, pH 8.0, $0.5 \mathrm{mM}$ EDTA). Purified DNA was checked by running it on a $0.8 \%$ agarose gel containing ethidium bromide. The DNAs were quantified using an Ultraspec-1100 pro UV-spectrophotometer (Amersham BioSciences, Hongkong, China). All the DNAs were diluted to a concentration of 20 $\mathrm{ng} / \mu \mathrm{l}$ in TE buffer.

\section{Bulk segregant analysis (BSA)}

Individual DNAs from the parents, their $\mathrm{F}_{1}$ progeny and high and low weight $\mathrm{F}_{2}$ and $\mathrm{BC}$ progeny separated on the basis of the weight of the cocoon and shell were screened. In the $F_{2}$, DNA from 10 individuals from each of the high and low weight groups were pooled and used for PCR. Similarly, DNA from pupae of the BC progeny were individually extracted, quantified, and diluted to a concentration of $20 \mathrm{ng} / \mu \mathrm{l}$.

\section{Primers and PCR}

A total of 240 EST SNP primers (forward and reverse) appropriate for the 28 linkage groups (Yamamoto et al., 2006, 2008), generously supplied by Insect Genome Research Group, National Institute of Agrobiological Sciences, Tsukuba, Japan, were used to amplify the DNA of parents and $F_{1}$ progeny. PCR reaction consisted of $20 \mathrm{ng}$ of template DNA, $0.2 \mathrm{mM}$ each of dATP, dCTP, dGTP, and dTTP (Fermentas Inc, Glen Burnie, $\mathrm{MD}$, USA), $1 \mu \mathrm{M}$ of each forward and reverse SNP primer, and $0.45 \mathrm{U}$ of Taq DNA polymerase (Fermentas ) in a $10 \mu \mathrm{l}$ volume. 35 cycles of PCR were performed on a PTC 200 Thermocycler Engine (Bio-Rad Laboratories, Hercules, CA, USA) as follows: $94^{\circ} \mathrm{C}$ for $2 \mathrm{~min}$, followed by 35 cycles of $94^{\circ} \mathrm{C}$ for $30 \mathrm{~s}, 60^{\circ} \mathrm{C}$ for $30 \mathrm{~s}$, and $72^{\circ} \mathrm{C}$ for $1 \mathrm{~min}$, with a final extension of $72^{\circ} \mathrm{C}$ for $1 \mathrm{~min}$. PCR products were analyzed by electrophoresis on $2 \%$ agarose gels and stained with ethidium bromide and visualised under UV light.

\section{RESULTS}

In the present study two strains of silkworm, Pure Mysore (PM) and CSR2, which differ in their silk yield traits, were used as parents. These two strains are very different in their commercial traits and characteristic features (Table 1). In the tropical conditions prevailing in India it is possible to rear six generations per year. March to August is an unfavourable period as the temperatures then are higher and larval duration is shorter, which results in the production of small cocoons. The period September to February is the most favourable period as the temperatures are optimum and the larval period is longer, which results in the production of large cocoons. Hence, for each of the different quantitative traits there is a range of values (Table 1). PM is a low yielding indigenous polyvoltine strain, which has been reared in south India for two hundred years. This strain is characterized by a short larval duration and low body, cocoon, and shell weights, with a low silk content, shorter filament length and poor quality silk. CSR2 is a bivoltine strain bred in the '90s from highly productive Japanese hybrids and, subsequently acclimatized to Indian tropical conditions, which was fixed by following appropriate breeding protocols. CSR2 takes longer to complete its larval development and has a higher body, cocoon, and shell weights, with a high raw silk yield, longer filament length and

TABLE 1. Characteristics of parental strains.

\begin{tabular}{lcc}
\hline Characteristics & Pure Mysore & CSR2 \\
\hline Larval markings & Plain & Plain \\
Cocoon colour and shape & Light greenish yellow spindle & White oval \\
Cocoon grains & Loose grains & Fine to medium \\
Percentage of pupation (\%) & $88.7-90.0$ & $75-80$ \\
Cocoon weight (g) & $0.800-1.100$ & $1.500-1.800$ \\
Shell weight (g) & $0.090-0.140$ & $0.350-0.400$ \\
Cocoon shell (\%) & $11-13$ & $22-24$ \\
Filament length (m) & $352-375$ & $800-1100$ \\
Filament size (denier*) & $1.9-2.0$ & $3.0-3.1$ \\
Raw silk (\%) & $8.0-8.6$ & $19.0-20.0$ \\
Neatness (points) & $76-80$ & $85-90$ \\
Reelability (\%) & $74-75$ & $80-85$ \\
Recommended season for rearing & All seasons & August-February \\
\hline
\end{tabular}

* Denier denotes weight in $\mathrm{g}$ of $9000 \mathrm{~m}$ of raw silk, which is a measure of filament size. 
TABLE 2. DNA polymorphism detected using parents and $F_{1}$ progeny.

\begin{tabular}{|c|c|c|c|c|}
\hline $\begin{array}{l}\text { Linkage } \\
\text { group }\end{array}$ & $\begin{array}{c}\text { No. of } \\
\text { primers } \\
\text { screened }\end{array}$ & $\begin{array}{l}\text { No. of } \\
\text { primers } \\
\text { indicating } \\
\text { similar } \\
\text { patterns }\end{array}$ & $\begin{array}{l}\text { No. of } \\
\text { primers } \\
\text { indicating } \\
\text { polymor- } \\
\text { phism }\end{array}$ & $\begin{array}{c}\% \text { of } \\
\text { polymor- } \\
\text { phism }\end{array}$ \\
\hline 1 & 4 & 3 & 1 & 25.0 \\
\hline 2 & 6 & 4 & 2 & 33.3 \\
\hline 3 & 19 & 15 & 4 & 21.1 \\
\hline 4 & 12 & 10 & 2 & 16.5 \\
\hline 5 & 8 & 6 & 2 & 25.0 \\
\hline 6 & 6 & 6 & 0 & 0.0 \\
\hline 7 & 12 & 12 & 0 & 0.0 \\
\hline 8 & 6 & 5 & 1 & 16.7 \\
\hline 9 & 5 & 1 & 4 & 80.0 \\
\hline 10 & 11 & 8 & 3 & 27.3 \\
\hline 11 & 9 & 4 & 5 & 55.6 \\
\hline 12 & 14 & 6 & 8 & 57.1 \\
\hline 13 & 18 & 16 & 2 & 11.1 \\
\hline 14 & 9 & 8 & 1 & 11.1 \\
\hline 15 & 11 & 11 & 0 & 0.0 \\
\hline 16 & 8 & 2 & 6 & 75.0 \\
\hline 17 & 6 & 6 & 0 & 0.0 \\
\hline 18 & 9 & 8 & 1 & 11.1 \\
\hline 19 & 7 & 7 & 0 & 0.0 \\
\hline 20 & 4 & 4 & 0 & 0.0 \\
\hline 21 & 9 & 9 & 0 & 0.0 \\
\hline 22 & 16 & 12 & 4 & 25.0 \\
\hline 23 & 4 & 4 & 0 & 0.0 \\
\hline 24 & 6 & 6 & 0 & 0.0 \\
\hline 25 & 3 & 3 & 0 & 0.0 \\
\hline 26 & 12 & 11 & 1 & 8.3 \\
\hline 27 & 3 & 2 & 1 & 33.3 \\
\hline 28 & 3 & 3 & 0 & 0.0 \\
\hline Total & 240 & 192 & 48 & 20.0 \\
\hline
\end{tabular}

better quality silk. It is evident from the table that the values for cocoon weight, shell weight, shell \%, filament length and raw silk \% for CSR2 are more than two fold greater than those of Pure Mysore.

\section{Detection of polymorphism between parents and $F_{1}$ progeny}

Two hundred and forty SNP primers representing all of the 28 linkage groups (LG) in silkworm were screened using the genomic DNA of the parents, PM and CSR2, and their $F_{1}$ progeny. Out of these, 192 primers resulted in similar patterns in the parents with no differences in the size of amplified products and hence the parents and $F_{1} s$ showed identical banding patterns (Table 2). A total of 48 primers yielded a distinct polymorphism between the parents showing clear size differences in the amplified products and the co-dominant expression of these polymorphic bands were observed in $F_{1}$, where two bands were detected. The highest polymorphism of $80 \%$ was found in LG 9, followed by LG $16(75 \%)$ and LG 12 $(57.1 \%)$. In the case of LGs $6,7,15,17,19,20,21,23$, 24,25 , and 28 , no polymorphism was recorded. The overall polymorphism was found to be $20 \%$. The details of primers showing distinct polymorphism between the parents are given in Table 3.

\section{Cocoon parameter data in parents, $F_{1}, F_{2}$ and $B C$ progeny}

In the mulberry silkworm, cocoon and shell weights are the two key traits that ultimately determine the yield of silk. Hence, in the present investigation, these two traits were selected for analysis. The two parents showing large differences in cocoon traits, viz., PM (low yielding), CSR2 (high yielding), and their $\mathrm{F}_{1}, \mathrm{~F}_{2}$ and $\mathrm{BC}$ progeny were reared simultaneously in the summer season of April-May 2010. After cocooning, the cocoon parameter data was recorded by sampling a total of 100 cocoons consisting of 50 females and 50 male cocoons of each of the parents and, $F_{1}$ and $F_{2}$ progeny. The mean and standard deviation of the cocoon and shell weights is listed in Table 4. PM and CSR2 differ in these two traits by more than a factor of two. In the $F_{1}$, cocoon and shell weights are $11 \%$ and $3 \%$ higher, respectively, than that of the high yielding parent CSR2, indicating over-dominance of the latter especially for cocoon weight. The frequency distributions of the cocoon and shell weights of 100 cocoons of $F_{2}$ was analyzed (Fig. 1). The cocoon weight ranged from $0.871 \mathrm{~g}$ to $1.800 \mathrm{~g}$, while the shell weight range between $0.129 \mathrm{~g}$ to $0.305 \mathrm{~g}$, with a nearly normal distribution, however, prominent shoulders at the lower weight ranges may be attributed to the micro-environmental differences in the trays in which the larvae of each batch were reared from hatching to cocooning. The mean values of the cocoon $(1.253 \mathrm{~g})$ and shell weights $(0.210 \mathrm{~g})$ for $F_{2}$ progeny is intermediate between that of the two parents (Table 4), due to the wide segregation of the parental genes for low and high values of cocoon traits. Based on cocoon weight, individuals with the top ten and bottom ten cocoon weights were selected from among the $F_{2}$ and BC progeny and bulked. The bulked samples also show more than two fold differences in their cocoon traits. The over-dominance of CSR2 is evident in the $\mathrm{BC}$ high value individuals, for which the values are much higher than for the $F_{2}$ high value individuals. The statistical analysis using Student's $t$-test also confirms there are highly significant differences between the parents and the bulked samples of the $\mathrm{F}_{2}$ and $\mathrm{BC}$ progeny.

\section{Identification of the DNA marker linked to cocoon traits using Bulked segregant analysis}

The genomic DNA of parents, PM, CSR2, their $\mathrm{F}_{1}$ progeny, $\mathrm{F}_{2}$ high and low bulks and, $\mathrm{BC}$ high and low bulks were amplified with the 48 informative SNP primer pairs, which revealed distinct polymorphism between the parents. Except for primer pair No. 04124 of LG 4, all the remaining 47 primer pairs revealed polymorphism between the parents and co-dominant expression in the $F_{1}$ as revealed by the 2 bands inherited from both parents. However, both the high and low bulks of $\mathrm{F}_{2}$ showed identical patterns with amplified products of the same size found in either PM or CSR2, indicating absence of linkage with the cocoon traits. In the case of SNP primer pair No. 04124 of LG 4, the low yielding parent PM showed an amplified product of $0.6 \mathrm{~kb}$ while the high yielding parent CSR2 had a heavier PCR product of 0.7 
TABLE 3. Details of SNP primers indicating polymorphism between parents.

\begin{tabular}{|c|c|c|c|c|}
\hline \multirow{2}{*}{ S1. no. } & \multirow{2}{*}{ Primer ID } & \multirow{2}{*}{$\begin{array}{c}\text { Linkage } \\
\text { group }\end{array}$} & \multicolumn{2}{|c|}{ Sequence } \\
\hline & & & Forward & Reverse \\
\hline 1 & 01065 & 1 & ACTGACGAGCTCCCTCTGAA & TTCTGTACGACAATACCGTTCG \\
\hline 2 & 02034 & \multirow{2}{*}{2} & TTTTTATTATATCACGTCCCC & CATAAGTTTGCGTGTAAAGC \\
\hline 3 & 02051 & & AGAAAATACTGTTACTCGCTAGG & CAAACCTAAAATGCAAATGG \\
\hline 4 & 03070 & \multirow{4}{*}{3} & TTCGAACCGTTTGTTGAAGTT & TGAACATGATTTTGACCCCC \\
\hline 5 & 03071 & & GGAAATTTACCCAATGACATGAA & TCGGCTAAGGTTTCAACCAA \\
\hline 6 & 03079 & & TCGTAATCGTCACTTTTAACCTC & GCACGTCTGGGTGTAGTTGA \\
\hline 7 & 03085 & & CGCAGCAAAATTAGGGTTGT & GCACTGCTCTTGCTAGGGTC \\
\hline 8 & 04110 & \multirow{2}{*}{4} & CTGTGCTGACTATCCGGTCA & CAACTATCAGTAAATGAAACCGACA \\
\hline 9 & 04124* & & CTCTCGATGCTTTCAGGACC & AAATTCGGTAGCCAGAGCCT \\
\hline 10 & 05053 & \multirow{2}{*}{5} & CATGGAAGGAAGAAAATACCCA & TGTTGTGTCAACGCCAATTC \\
\hline 11 & 05106 & & GCAAACCTCGAAATCCGATA & GTTATGTAACCCGTGTGGGC \\
\hline 12 & 08047 & 8 & CATGATAGGAGAGTTATAGCGTCG & CCTCCCTTCAAGCCGAAAC \\
\hline 13 & 09075 & \multirow{4}{*}{9} & TGGAATGTTTAAATGCACGC & GTTTGTTACAGAGCGCGGTT \\
\hline 14 & 09080 & & AACCGCCAATTTAACGTCAC & ATGTTTCCCGAATCTGGTTG \\
\hline 15 & 09082 & & GGTTGCATAACTAAGCTGCG & TTGGCGTGATTCTTAATACCG \\
\hline 16 & 09083 & & TGGCCAAATTGGGAATTTAC & САТСАТСТTСАТСАТССТСАТСА \\
\hline 17 & 10098 & \multirow{3}{*}{10} & CGAATACGCATCGTGTCAAA & TGCAACGGTCTTAAATGAGG \\
\hline 18 & 10105 & & TCGCGATGTGAGTAATGGTG & CATGACATATTCGCAAAACG \\
\hline 19 & 10112 & & CTGATCATAGCTCTGTAATTATTATGC & TAGATTCAGAAACGGGCCAC \\
\hline 20 & 11049 & \multirow{5}{*}{11} & ACACGTGTCCAGTGTTGCAT & TCGATATCGGAGGTTCGAGT \\
\hline 21 & 11075 & & TGGCACCTTAAAGTTACTGG & CAAACATGTGTTCAAAGTCG \\
\hline 22 & 11092 & & TAAACAGTTCGTTTGCCGAA & AGTAAACGCATATTGCCCCC \\
\hline 23 & 11105 & & TGTCTTCCTCTGGAGGGTTG & TCCATTTATCACTTCATTACAAATCA \\
\hline 24 & 11108 & & TGGGATAAGGTGGAGAAAAAGA & GTTCCACGGGGAGCAACTAT \\
\hline 25 & 12047 & \multirow{8}{*}{12} & AGTGTCCTTTGTCATTCTGG & CCAGTTAAGCATTCATAGCC \\
\hline 26 & 12059 & & GCTGTGTCTGATTAGTGCCG & GCAAGCAACAATGTTCCAAA \\
\hline 27 & 12073 & & CGGTGGAAAATTAAACTTATGGG & CGACTAGTAGTTTCTTTAGTTTTCGC \\
\hline 28 & 12077 & & CAATGAAGAAGTTTGAAGGGCT & TTCTCGTAAAATTATTCATCAAAACA \\
\hline 29 & 12079 & & AAACTAAGCTTTTGGGTCCGA & GCGCCATTCTAAGTGACCAT \\
\hline 30 & 12080 & & AAATTGCGAGCTTGACACG & AAGCGGAGGCTCTGGAAT \\
\hline 31 & 12081 & & TGGCTTCATTCTAGAAGACATTTTT & TGAAGTTTTCAATGCAACCAA \\
\hline 32 & 12082 & & TCGACGGCTTACTTAGTGAGTG & ATACCTCTCAGTGGGCAACG \\
\hline 33 & 13129 & \multirow{2}{*}{13} & CCAAACATTTCTTTGGTCGG & GGTCTCAAGTATAGTTACAACGGATG \\
\hline 34 & 13138 & & TTACGTAGCGCGTCAAAATC & TTAAGATTGAAGATGGAAGATAGAGA \\
\hline 35 & 14050 & 14 & TGTAGAGTAACCGACTATGATGATGA & TTTCAACTAGCAGTTCGCTATCTT \\
\hline 36 & 16006 & \multirow{6}{*}{16} & GTGGTCTTCCTCCCAGACAA & CGATCATGCCACTCTGTAAA \\
\hline 37 & 16015 & & GACTCTGGTACCTGGATGTGG & TCGTAGACGACTTAAAAGTTCCG \\
\hline 38 & 16035 & & CGTTACTAAAAGCCTCCTCCG & AACGAGATCATACGCCAAAAA \\
\hline 39 & 16057 & & AACGAGATCATACGCCAAAAA & GCTTGAGACGGGTGAAGAAT \\
\hline 40 & 16058 & & CTCCCGTCTACAACGCTTTT & TCGAGTACGCATATCCTAATTATACG \\
\hline 41 & 16059 & & CAAGGATAAAGAAGAAGAAACTCACC & TGTCATTCCAGCATCTTGGT \\
\hline 42 & 18086 & 18 & GACCCACTGAGAAGATCCCA & CGTTTCATCAATTTCACAGAACA \\
\hline 43 & 22054 & \multirow{4}{*}{22} & AAACACAACTTGGCCTCCAC & AAAGGGAGTCCTGGCTTAGA \\
\hline 44 & 22075 & & CCAGAGATCATTTTTGCAACTT & GGGCCCATTCCTACTGAAA \\
\hline 45 & 22090 & & CCGTAGCGTAACAAACGACA & CGATATAGGCTTCACAGGTGTTT \\
\hline 46 & 22094 & & GACAGCTGCCCGAGCTATTA & CAGTTGCTAGGCAAAGAGCA \\
\hline 47 & 26048 & 26 & TCCAACCCCACTAAATTTGC & GAATCTTAGGTTTCAAATTAGGCA \\
\hline 48 & 27044 & 27 & TTTAGGCACTACAAGCACCG & CACAGAAACGCCAAGCATT \\
\hline
\end{tabular}

* Primer pair showing linkage with cocoon traits.

$\mathrm{kb}$ (Fig. 2). The sequences of this primer pair are as follows. F: CTCTCGATGCTTTCAGGACC and R: AAATTCGGTAGCCAGAGCCT. In the $\mathrm{F}_{1}$, co-dominant expression of two bands of $0.6 \mathrm{~kb}$ and $0.7 \mathrm{~kb}$ was observed. In the high bulk of $\mathrm{F}_{2}, 0.7 \mathrm{~kb}$ product found in CSR2 was present, while in the low bulk, a $0.6 \mathrm{~kb}$ band specific to PM was amplified. To confirm these results, the DNA profiles of high bulk and low bulk individuals of backcrosses, namely, $(\mathrm{PM} \times \mathrm{CSR} 2) \times \mathrm{CSR} 2$ and $(\mathrm{PM}$ $\times$ CSR2) $\times$ PM, respectively were analyzed by amplifying with primer pair No. 04124 (Fig. 3). The $0.7 \mathrm{~kb}$ product specific to the CSR2 parent was found in all the high bulk individuals and the $0.6 \mathrm{~kb}$ band of the low yielding parent, $\mathrm{PM}$, in all the individuals in the low value bulk 
TABLE 4. Mean values with standard deviations and $t$ values for cocoon traits of parents, $\mathrm{F}_{1}, \mathrm{~F}_{2}$ and $\mathrm{BC}$ progeny.

\begin{tabular}{|c|c|c|c|c|c|}
\hline Sl. no. & Parents / Progeny & Cocoon weight $(\mathrm{g})$ & $t$ value & Shell weight $(\mathrm{g})$ & $t$ value \\
\hline 1 & Pure Mysore $\left(\mathrm{P}_{1}\right)$ & $0.751 \pm 0.095$ & $24.3 * * *$ & $0.098 \pm 0.016$ & $15.05 * * *$ \\
\hline 2 & $\operatorname{CSR} 2\left(\mathrm{P}_{2}\right)$ & $1.535 \pm 0.037$ & & $0.264 \pm 0.031$ & \\
\hline 3 & $\mathrm{PM} \times \mathrm{CSR} 2 \mathrm{~F}_{1}$ & $1.708 \pm 0.189$ & & $0.273 \pm 0.022$ & \\
\hline 4 & $\mathrm{PM} \times \mathrm{CSR} 2 \mathrm{~F}_{2}($ Mean $)$ & $1.253 \pm 0.192$ & & $0.210 \pm 0.032$ & \\
\hline 5 & $\mathrm{PM} \times \mathrm{CSR} 2 \mathrm{~F}_{2}($ High bulk $)$ & $1.601 \pm 0.104$ & $18.5^{* * *}$ & $0.274 \pm 0.017$ & $16.21 * * *$ \\
\hline 6 & $\mathrm{PM} \times \mathrm{CSR} 2 \mathrm{~F}_{2}($ Low bulk $)$ & $0.950 \pm 0.040$ & & $0.159 \pm 0.015$ & \\
\hline 7 & $(\mathrm{PM} \times \mathrm{CSR} 2) \times \mathrm{CSR} 2 \mathrm{BC}($ High bulk $)$ & $1.855 \pm 0.119$ & $30.6^{* * *}$ & $0.332 \pm 0.030$ & $16.15^{* * *}$ \\
\hline 8 & $(\mathrm{PM} \times \mathrm{CSR} 2) \times \mathrm{PM} \mathrm{BC}($ Low bulk $)$ & $0.928 \pm 0.094$ & & $0.145 \pm 0.021$ & \\
\hline
\end{tabular}

Explanations: In the case of parents, $F_{1}$ and $F_{2}$ means, the values of cocoon and shell weights are mean values for 100 cocoons made up of equal numbers of cocoons of both sexes ( 50 females and 50 males). For $\mathrm{F}_{2}$ and $\mathrm{BC}$ bulk samples, the mean values are for samples of 10 cocoons. $t$ values for the Student's $t$-tests of the means of cocoon and shell weights of the parents (S1. no. 1 versus 2 ), $\mathrm{F}_{2}$ bulk samples (Sl. no. 5 versus 6), and BC bulk samples (Sl. no. 7 versus 8 ). $* * *$ denotes significance at $0.001 \%$.

samples. These results clearly indicate that the DNA regions amplified by primer pair No. 04124 of LG 4 are closely linked to quantitative trait loci (QTL) controlling cocoon traits.

\section{DISCUSSION}

To identify a DNA marker closely linked to a gene or genomic region, two methods have proved effective in selecting from a large number of markers, the few that reside near a targeted locus. One involves using near isogenic lines (NILs), which are developed by transferring the target gene from the donor parent (DP) into a recurrent parent (RP) by repeated backcrossing of DP for 5-6 generations, simultaneously selecting the target gene in every generation. The resultant line is known as NIL, which is nearly isogenic with the RP as most of its genome is derived from RP along with a small portion of the genome of DP flanking the target gene. Any DNA polymorphism between the DP and NIL is likely to be linked to the target gene (Young \& Tanksley, 1989). Using this method, very active digestive amylase genes from Indian polyvoltine strains used as DPs were introgressed into the genetic background of high yielding bivoltine strains of temperate origin with a "null" type of amylase and NILs were developed with a DP type of amylase (Ashwath et al., 2001). Using these NILs, RAPD markers linked to the high activity digestive amylase genes in silkworm were identified (Ashwath et al., 2010)

The other method, which is more generally used, is referred to as bulked segregant analysis (BSA), which relies on the use of segregating populations of $F_{2}$. This method involves comparing two pooled DNA samples of individuals from a segregating $F_{2}$ population originating from a single cross. Initially, $\mathrm{F}_{1} \mathrm{~S}$ of parents with contrasting phenotypes of the target trait (e.g. high and low or resistant and susceptible) and $\mathrm{F}_{2}$ progeny are generated. Using the selected informative DNA markers, which show distinct polymorphism between the parents, the DNA profile of the parents, $F_{1}$ progeny and $F_{2}$ bulks segregating for the target trait (high and low or resistant and susceptible) are then compared. The DNA markers that are polymorphic between the bulks are likely to be linked to the target gene.

BSA has several advantages over the use of nearisogenic lines to identify markers in specific regions of the genome. Genetic walking will be possible by multiple rounds of BSA; each new pair of bulk samples will differ at a locus identified in the previous round of the analysis. This approach has widespread application both in those species where selfing is possible and those that are obligatorily outbreeding (Michelmore et al., 1991). Further, the simplicity and low cost of BSA have led to its use for

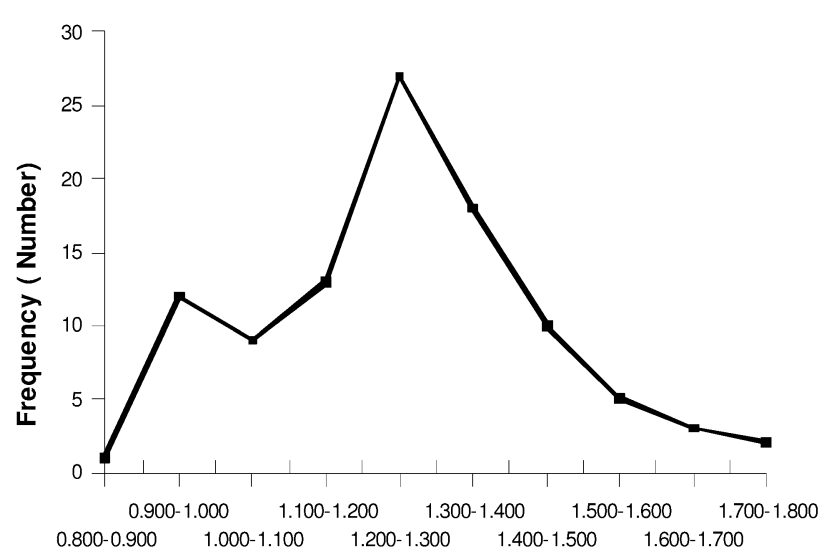

a

Cocoon weight (g)

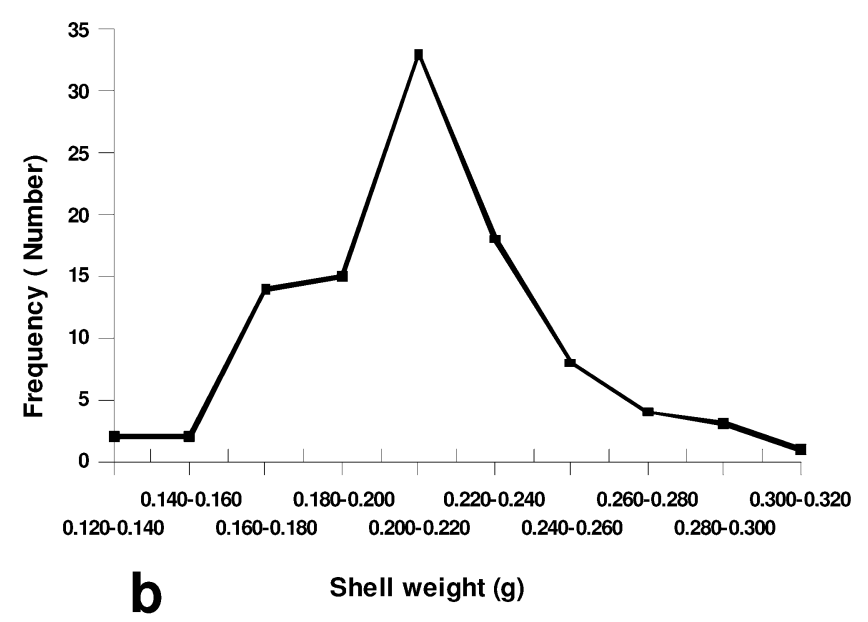

Fig. 1. Frequency of cocoon weights (a) and shell weights (b) recorded for $F_{2}$ progeny. Note the nearly normal distribution pattern recorded for samples of 100 cocoons. 


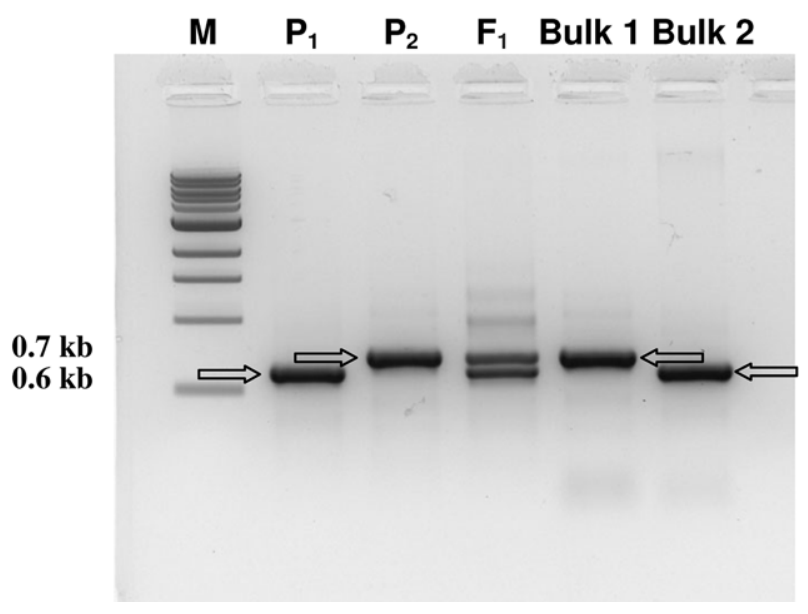

Fig. 2. Amplification profiles of SNP primer pair No. 04124 of linkage group 4 of parents, $F_{1}$ and $F_{2}$ bulk samples. Arrows indicate polymorphic SNP products of $0.6 \mathrm{~kb}$ in $\mathrm{P}_{1}$ (Pure Mysore, low yielding strain) and $0.7 \mathrm{~kb}$ in $\mathrm{P}_{2}$ (CSR2, high yielding strain). $\mathrm{F}_{1}$ shows co-dominant expression of both products revealed by two bands. Bulk 1 (high cocoon weight bulk of $F_{2}$ ) shows the $0.7 \mathrm{~kb}$ product of high yielding parent $\mathrm{P}_{2}$, while Bulk 2 (low cocoon weight bulk of $F_{2}$ ) has the $0.6 \mathrm{~kb}$ band of low yielding parent $\mathrm{P}_{1}$. M denotes the DNA size marker.

more complex traits, including traits whose genetic control is unknown. This method is often restricted to segregating generations, which are simple and cheap to produce, such as the backcross $(\mathrm{BC})$ and $\mathrm{F}_{2}$ generations. For dominant marker systems, the $\mathrm{F}_{2}$ is considered to be the best generation on which to conduct a BSA (Mackay \& Caligari, 2000).

SNP primers supplied by Silkworm Genome Research Group Tsukuba, Japan were used to detect polymorphism between the two strains viz Pure Mysore and CSR2, both of which are very widely reared but differ greatly in the quality and amount of silk they produce. Initially the template DNAs of parents and $F_{1}$ progeny were amplified using 240 SNP primer pairs belonging to all the 28 linkage groups in silkworm. Out of these 240 primer pairs, 48 revealed distinct polymorphism between the parents, which was confirmed by the co-dominant expression of both the polymorphic products in the $F_{1}$. The overall polymorphism was found to be $20 \%$. This low level of polymorphism may be attributed to the fact that the silkworm strains used in the present study differ from those used in the original SNP studies of Yamamoto et al. (2006), who used their own standard strains, p50T and C108T, which are used routinely for molecular linkage map construction. In addition, in the present study only deletions or insertions could be detected, which would result in different sized amplified products, but nucleotide substitutions are unlikely to affect DNA fragment migration in agarose gels, which may escape detection even if they are polymorphic. The original studies also used sequence based methods that detect nucleotide substitutions.

Yamamoto et al. (2006) have constructed a linkage map of 534 SNPs based on the bacterial artificial chromosome (BAC) end sequences of the silkworm using the male

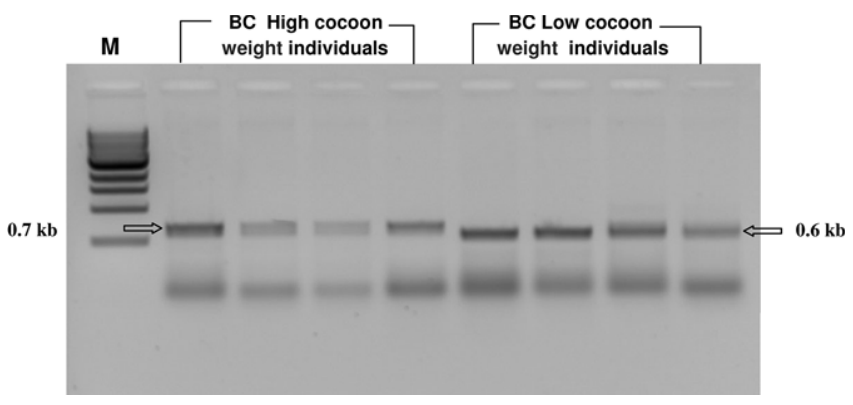

Fig. 3. DNA profiles of the high and low cocoon weight bulk samples of the backcross progeny amplified with SNP primer pair No. 04124 of linkage group 4. Note the presence of $0.7 \mathrm{~kb}$ fragment of high yielding parent $\mathrm{P}_{2}$ in the high cocoon weight samples of backcross $(\mathrm{BC})$ progeny $(\mathrm{PM} \times \mathrm{CSR} 2) \times \mathrm{CSR} 2$ and $0.6 \mathrm{~kb}$ band of low yielding parent $\mathrm{P}_{1}$ in the low cocoon weight individuals of $\mathrm{BC}$ progeny $(\mathrm{PM} \times \mathrm{CSR} 2) \times \mathrm{PM}$. M denotes the DNA size marker.

backcross population of standard strains, p50T and C108T, and this map was subsequently extended to 1755 SNPs (Yamamoto et al., 2008). However, out of the 240 SNP primer pairs used in the present study, only 58 SNP markers were located in the linkage map and of these only 11 revealed polymorphisms between the parents.

The 48 informative SNP primer pairs, which revealed a distinct polymorphism between the parents, were used for amplifying the genomic DNA of parents, $F_{1}$ and two bulk samples of high and low cocoon traits, and of the 48 primer pairs, only one, namely No. 04124 pertaining to LG 4 , revealed a clear polymorphism between the $F_{2}$ bulk samples of high and low cocoon traits, which indicates a linkage between the DNA segments amplified by this primer of LG 4 and the genes controlling cocoon traits.

Lu et al. (2004) identified eleven QTLs using a backcrossed population derived from a cross between C100 and Dazao, and using the AFLP technique mapped two QTLs for whole cocoon weight on LGs 6 and 19; three QTLs for cocoon shell weight on LG 3, 14, and 19; three QTLs for cocoon shell ratio on LGs 2, 14, and 19. Recently, Zhan et al. (2009) constructed an integrated map of SSR markers using two populations of four silkworm strains, Nistari (Indian origin), Fa50B (French origin) and Jingsong and Lan 10 (Chinese origin). They identified six QTLs involved in cocoon weight (CW), cocoon shell weight (CSW), cocoon shell ratio (CSR), and pupal weight (PW). Three QTLs (Q1, Q2, Q3) for CW were located in the LGs 1, 21, and 23, while two QTLs (Q1 and Q4) for CSW were found in linkage groups 1, 22, and 23. It is interesting to note that Q1 of LG 1 has simultaneous effects on CW, CSW, and PW, indicating that the same QTL may be controlling all these traits. Lie et al. (2010) carried out a composite interval mapping of QTLs employing AFLP markers using the backcross between Dazao and C100 strains and located 19 putative QTLs including 5 QTLs (in LGs 4, 10,11,15 and, 33) for cocoon weight, 4 QTLs (in LGs 4, 10,12 and, 33) for cocoon shell weight, 6 QTLs (in LGs 4, 10,11,15 and, 33) for pupa weight and 4 QTLs (in LGs 6,12,21 and, 23) for cocoon shell ratio. All these studies have 
indicated the possibility of the presence of a QTL for CW and CSW in the LGs 1, 3, 4, 6, 10, 11, 12, 14, 15, 19, 21, 22 , and 23. However, in the current study 240 primer pairs screened among the 28 LGs did not reveal polymorphism in LGs $6,15,19,21$, and 23, which are reported to carry QTL and hence even if a major QTL for CW and $\mathrm{CSW}$ segregated in the $\mathrm{F}_{2}$ or $\mathrm{BC}$, there was no way of detecting it. In the present analysis, the SNP primer pair No. 04124 revealed linkage with both cocoon and shell weights. It is well established that both $\mathrm{CW}$ and CSW are strongly positively correlated (Chatterjee et al., 1993; Zhan et al., 2009) and the genetic relatedness of these traits is corroborated by the present finding, which indicates the possibility of a QTL in LG 4, which controls both these traits. Pradeep et al. (2007) have also revealed the pleiotropic role of one ISSR marker significantly associated with many biomass traits like larval weight, cocoon weight, shell weight, shell ratio, and floss content.

The strength of the BSA used in the present study is only sufficient to detect tightly linked markers because crossing over generates a heterozygous pool in the $F_{2}$ and the weakness of this method is lack of statistical power as it is not be possible to know how many individuals contribute to each of the co-dominant markers in a pooled $\mathrm{F}_{2}$ sample scored as heterozygous using PCR. Hence, additional QTLs contributing to a trait may be missed. But the purpose of this study was to detect tightly linked molecular markers and hence the use of the BSA method, whereas others have used map based approaches, which can detect more of the QTLs contributing to the trait in question.

The sex chromosome mechanism in the mulberry silkworm is of the ZZ : WZ type, with males having ZZ chromosomes (homogametic) and females the heterogametic sex with WZ chromosomes. The Z-linked locus, Lm (late maturity gene) is well known to prolong larval duration, which in turn increases the expression of genes (Lie et al., 2010) controlling larval weight, cocoon weight, shell weight etc., while its allele, $\mathrm{Lm}^{e}$ (early maturity) decreases larval duration, thereby resulting in low cocoon weights. The silkworm strains of temperate origin (like CSR2 used in the present study) have the $L m$ allele and produces more silk than those of tropical origin (Pure Mysore), which have the $L m^{e}$ allele. However, in the present investigation, only one polymorphic marker for the $\mathrm{Z}$ chromosome was found and it did not appear to be associated with the cocoon traits.

It is interesting to note that Lie et al. (2010) have also found QTLs for CW and CSW in the LG 4 using one of the silkworm strains of Indian origin, namely, Nistari, which is an indigenous low yielding polyvoltine strain popular in North-Western India. In the present study, the Indian low yielding polyvoltine strain, Pure Mysore was used, which is widely cultivated in Southern India. These two Indian silkworm strains are low yielding but survive better in an Indian tropical climate, features which are likely to have evolved over the past 200 years of their cultivation for silk production. The results of the present study and those of Lie et al. (2010) indicate the possibility that the genomic regions in the LG 4 controlling cocoon traits may be conserved in the Indian silkworm strains, Nistari and Pure Mysore during the course of domestication for the last 200 years.

Though there are few articles on interval mapping for QTL analysis of economic traits in silkworm using DNA markers like SSRs and AFLPs, this is the first report of using SNPs with BSA to identify a QTL.

\section{CONCLUSION}

Though India is today the second largest producer of silk in the world, most of the silk comes from crosses between indigenous polyvoltines and temperate bivoltine strains and the quality of the silk is not of international grade. There is a need to improve the cocoon traits of Indian silkworm strains by using both conventional and molecular methods. In an attempt to achieve this, DNA markers linked to cocoon traits, which play a vital role in determining the quality and quantity of silk, were identified. Bulked segregant analysis using SNP primers revealed large differences in the cocoon traits of the parents, their $F_{1}$ and bulked samples of $F_{2}$ and $B C$ progeny, which clearly indicate linkage of the DNA segments amplified by SNP primer pair No. 04124 to linkage group 4 , with the QTL governing cocoon and shell weight characters. The results of the present study indicate that it may be possible to use SNP markers in silkworm breeding programs aimed at improving the cocoon traits of low yielding polyvoltine strains of Indian silkworms, which could potentially greatly increase silk yield and quality. The QTLs of the mulberry silkworm have been analyzed by various research groups using different molecular markers, like SSRs, AFLPs and SNPs on diverse silkworm strains, which has indicated close association of various linkage groups with cocoon traits. Hence there is a need for the development of a saturated linkage map integrating all the mapped molecular markers for locating chromosome segments tightly linked to economic traits so that marker assisted selection can be successfully employed to quickly and precisely improve traits of interest in silkworms.

ACKNOWLEDGEMENTS. The authors convey their grateful thanks to the research group of the National Institute of Agrobiological Sciences, Japan, especially K. Yamamoto, K. Mita, and K. Kadono-Okuda for generously supplying the SNP primers. M. Slathia is grateful for a DBT fellowship. Financial support from the Department of Biotechnology, Govt of India, New Delhi is gratefully acknowledged.

\section{REFERENCES}

Ashwath S.K., Morrison M.N. \& Datta R.K. 2001: Development of near isogenic lines of productive silkworm breeds by isozyme marker based selection. Proc. Nat. Acad. Sci. India (B) 71: 207-222.

Ashwath S.K., Sreekumar S., Toms J.T., Dandin S.B. \& Kamble C.K. 2010: Identification of DNA markers linked to digestive amylase genes in the mulberry silkworm, Bombyx mori. J. Insect Sci. 10: doi: 10.1673/031.010.8401

Baxter S.W., Papa R., Chamberlain N., Humphray S.J., Joron M., Morrison C., Ffrench-Constant R.H., McMillan W.O. 
\& JigGins CD. 2008: Convergent evolution in the genetic basis of Müllerian mimicry in heliconius butterflies. Genetics 180: $1567-1577$.

Bradley K.M., Elmore J.B., Breyer J.P. Yaspan B.L., Jessen J.R., KNAPIK E.W. \& SMITH J.R. 2007: A major zebrafish polymorphism resource for genetic mapping. Genome Biol. 8: R55.

Chantret N., Sourdille P., Roder M., Tavaud M., Bernard M. \& Doussinault G. 2000: Location and mapping of the powdery mildew resistance gene MIRE and detection of resistance QTL by Bulked Segregant Analysis (BSA) with microsatellites in wheat. Theor. Appl. Genet. 100: 1217-1224.

Chatterjee S.N., Rao C.G.P., Chatterjee G.K., Ashwath S.K. \& PatnaiK A.K. 1993: Correlation between yield and biochemical parameters in the mulberry silkworm, Bombyx mori L. Theor. Appl. Genet. 87: 385-391.

Gadau J., Gerloff C.U., Krugen N., Chan H., Schmid-Hempel P., Wille A. \& Page R.E. 2001: A linkage analysis of sex determination in Bombus terrestris (L.) (Hymenoptera: Apidae). Heredity 87: 234-242.

Govindaraj P., Arumugachamy S. \& Maheswaran M. 2005: Bulked segregant analysis to detect main effect QTL associated with grain quality parameters in Basmati 370/ASD 16 cross in rice (Oryza sativa L) using SSR markers. Euphytica 144: $61-68$.

KanG S.T. \& Mian M.A. 2010: Genetic map of the powdery mildew resistance gene in soybean PI 243540. Genome 53: 400-405.

Khamnamtong B., Thumrungtanakit S., Klinbunga S., Aoki T., Hirono I. \& Menasveta P. 2006: Identification of sex-specific expression markers in the giant tiger shrimp (Penaeus monodon). J. Biochem. Mol. Biol. 39: 37-45.

Kaiser M.G., Deeb N. \& Lamont S.J. 2002: Microsatellite markers linked to Salmonella enterica serovar enteritidis vaccine response in young $\mathrm{F}_{1}$ broiler-cross chicks. Poultry Sci. 81: 193-201.

Lie Z., Cheng L., Fang-Yin D. \& Show-Min F. 2010: Mapping of major quantitative trait loci for economic traits of silkworm cocoon. Gen. Mol. Res. 9: 78-88.

Lu C., Li B., Zhaо A. \& XIANG Z. 2004: QTL mapping of economically important traits in silkworm (Bombyx mori). Sci. China C Life Sci. 47: 477-484.

Mackay L.J. \& Caligari P.D.S. 2000: Efficiencies of $F_{2}$ and backcross generations for bulked segregant analysis using dominant markers. Crop Sci. 40: 626-630.
Michelmore R.W., Paran I. \& Kesseli R.V. 1991: Identification of markers linked to disease-resistance genes by bulked segregant analysis: A rapid method to detect markers in specific genomic regions by using segregating populations. Proc. Natl. Acad. Sci. USA 88: 9828-9832.

Poulsen D.M.E., Henry R.J., Johnson R.P., Irwin J.A.G. \& REesE R.G. 1995: The use of bulk segregant analysis to identify a RAPD marker linked to leaf rust resistance in barley. Theor. Appl. Genet. 91: 270-273.

Pradeep A.R., Jingade A.H. \& URS R.S. 2007: Molecular markers for biomass traits: association, interaction and genetic divergence in silkworm Bombyx mori. Biomark Insights 2: 197-297.

Severson D.W., Zaitlin D. \& Kassner V.A. 1999: Targeted identification of markers linked to malaria and filarioid nematode parasite resistance genes in the mosquito Aedes aegypti. Genet. Res. 73: 217-224.

Wang D, Mao H.L., Chen H.X., Liu H.Q. \& Gui J.F. 2009: Isolation of Y- and X-linked SCAR markers in yellow catfish and application in the production of all-male populations. Anim. Genet. 40: 978-981.

Werner S., Diederichsen E., Frauen M., Schondelmaier J. \& JuNG C. 2008: Genetic mapping of clubroot resistance genes in oilseed rape. Theor. Appl. Genet. 116: 363-372.

Yamamoto K., Narukawa J., Kadono-OKuda K., Narukawa J., Sasanuma M., Sasanuma S., Minami H., Shimomura M., Suetsugu Y., Banno Y., Osoegawa K., De Jong P.J., GoldSмIтH M.R. \& MiтA K. 2006: Construction of a single nucleotide polymorphism linkage map for the silkworm, Bombyx mori, based on bacterial artificial chromosome end sequences. Genetics 173: 151-161.

Yamamoto K., Nohata J., Kadono-Okuda K., Nohata J., Sasanuma M., Suetsugu Y., Banno Y., Fuji H., Goldsmith M.R. \& MiтA K. 2008: A BAC-based integrated linkage map of the silkworm Bombyx mori. Genome Biol. 28: R21.

Young N.D. \& TANKSLEY S.D. 1989: RFLP analysis of the size of chromosomal segments retained around the $\mathrm{Tm}-2$ locus of tomato during backcross breeding. Theor. Appl. Genet. 77: 353-359.

Zhan S., Huang J., Guo Q., Zhao Y., Li W., Miao X., GoldSMITH M.R., Li M. \& HuANG Y. 2009: An integrated genetic linkage map for silkworms with three parental combinations and its application to the mapping of single genes and QTL. BMC Genomics 10: 389.

Received December 15, 2010; revised and accepted March 11, 2011 